\title{
Variations with modest effects have an important role in the genetic background of type 2 diabetes and diabetes-related traits
}

Hayato Fujita, Kazuo Hara, Nobuhiro Shojima, Momoko Horikoshi, Minoru Iwata, Yushi Hirota, Kazuyuki Tobe, Susumu Seino and Takashi Kadowaki

Journal of Human Genetics (2012) 57, 809; doi:10.1038/jhg.2012.132

Correction to: Journal of Human Genetics (2012) 57, 776-779;

doi:10.1038/jhg.2012.110; published online 20 September 2012

In the version of this article initially published, spelling of Yushi Hirota was wrong. The error has been corrected and the online HTML and PDF versions now carry the correct name. 